\title{
50 Jahre Zeitschrift WIRTSCHAFTSINFORMATIK: Auf zu neuen Ufern...
}

\author{
DOI 10.1007/s11576-008-0110-5
}

\section{Der Autor}

Prof. Dr. Hans Ulrich Buhl

Universität Augsburg

Lehrstuhl für Betriebswirtschaftslehre,

Wirtschaftsinformatik, Informations-

und Finanzmanagement

Universitätsstraße 16

86135 Augsburg

Deutschland

hans-ulrich.buhl@wiwi.uni-augsburg.de

This article is also available in English via http://www.springerlink.com and http://www.bise-journal.org: Buhl HU (2008) 50 Years of WIRTSCHAFTSINFORMATIK: Moving on to New Shores.... Bus Inf Syst Eng. doi 10.1007/ s12599-008-0006-3.
Vor 50 Jahren - im Jahr 1959 - wurde erstmalig die Zeitschrift elektronische datenverarbeitung herausgegeben mit dem Ziel, die existierende Lücke im Bereich der Fachveröffentlichungen „über die praktische Anwendung von Rechenanlagen in der Wirtschaft" (o. V. 1959, S. 3) zu schließen. Damals ahnten vermutlich weder Redaktion noch Verlag noch Leser, dass diese sich im Lauf von fünf Jahrzehnten unter dem Namen WIRTSCHAFTSINFORMATIK zur bedeutendsten wissenschaftlichen Zeitschrift des - damals noch in den Kinderschuhen steckenden - gleichnamigen Fachs im gesamten deutschsprachigen Raum, zu dessen zentralem Organ und zur einzigen international anerkannten deutschsprachigen Zeitschrift des Fachs entwickeln würde. Diesen Erfolg - und damit auch den 50. Geburtstag der Zeitschrift - wollen wir mit diesem Jubiläumsheft gebührend feiern.

Was liegt näher, als auf Basis der eben skizzierten Erfolge und anlässlich dieses Jubiläums die strategischen Weichen zu stellen, um die Zeitschrift vor dem Hintergrund der an Bedeutung gewinnenden Internationalisierung der Forschung evolutionär weiterzuentwickeln, der gestaltungsorientierten Wirtschaftsinformatik-Forschung eine einzigartige globale Plattform zu bieten und dadurch sämtliche technoökonomisch orientierten Leser und Autoren zu anzusprechen?

Diesem Heft kommt eine Doppelfunktion zu: Es ist zugleich Jubiläumsheft und Erstausgabe einer umfassenden, zweisprachigen, Wissenschaftler wie Praktiker adressierenden Triple-Strategie, die von den Herausgebern und dem Verlag in den letzten beiden Jahren konzipiert und implementiert wurde. Ab diesem Heft erscheint parallel und inhaltlich identisch zu einer weiterentwickelten WIRTSCHAFTSINFORMATIK und in der Verantwortung eines stark erweiterten internationalen Herausgeberkreises ein englischsprachiges und über SpringerLink beziehbares E-Journal unter dem Namen „Business \& Information Systems Engineering (BISE) - The International Journal of WIRTSCHAFTSINFORMATIK". Zusätzlich bringt der Gabler-Verlag mit Unterstützung des wissenschaftlichen Herausgeberkreises eine deutschsprachige, auf die Bedürfnisse der Wirtschaftsinformatik-Praktiker zugeschnittene Zeitschrift unter dem Namen Wirtschaftsinformatik \& Management (WUM) heraus.

Mit diesem Jubiläumsheft möchten wir dazu beitragen, durch ein hochselektives Spektrum ausgewählter Beiträge die dynamische Entwicklung der Zeitschrift, des Fachs und der Implikationen auf Wissenschaft und Praxis über die letzten fünf Jahrzehnte facettenreich darzustellen und durch diese Perspektive der letzten 50 Jahre auch zukunftsgerichtete, richtungweisende Impulse zu geben.

Zu Beginn finden sich zwei Beiträge zur Entwicklung der Zeitschrift. So diskutieren zum einen Norbert Szyperski, Peter Mertens und Wolfgang König als ehemalige Geschäftsführende Herausgeber und Interviewpartner die wesentlichen Entwicklungen ihrer jeweiligen Herausgeberzeit aus heutiger Perspektive. Zum anderen analysieren Ulrich Hasenkamp und Peter Stahlknecht die wichtigsten Entwicklungslinien und inhaltlichen Schwerpunkte der Zeitschrift.

Dem folgen sieben eingeladene Beiträge ausgewiesener Persönlichkeiten aus Wissenschaft und Praxis. Hermann-Josef Lamberti und Matthias Büger erörtern am Beispiel der Deutschen Bank AG die Erkenntnisse des IT-Einsatzes in Banken angefangen bei Lochkartenmaschinen bis hin zur Industrialisierung und Auslagerung von IT-Dienstleistungen. Martin Jetter, Gerhard Satzger und Andreas Neus zeigen am Beispiel der Transformation der IBM Corporation zu einem global integrierten und dienstleistungsorientierten Unternehmen, welche Auswirkungen IKT-basierte Innovationen auf Geschäftsmodell, Organisation und Unternehmenskultur haben. Günter Müller rekapituliert die Entstehung des Internets, sucht nach Analogien zur Entste- 


\begin{tabular}{|c|c|c|c|c|}
\hline Rubrik & Autor(en) & Titel & $\begin{array}{l}\text { Jahr der Erst- } \\
\text { veröffentlichung }\end{array}$ & $\begin{array}{l}\text { Nominierende( } r) \\
\text { Herausgeber }\end{array}$ \\
\hline WI - Aufsatz & Erwin Grochla & $\begin{array}{l}\text { Betriebsinformatik und Wirtschaftsinformatik } \\
\text { als notwendige anwendungsbezogene } \\
\text { Ergänzung einer allgemeinen Informatik }\end{array}$ & 1969 & $\begin{array}{l}\text { Ulrich Hasenkamp, } \\
\text { Norbert Szyperski }\end{array}$ \\
\hline WI - Aufsatz & Henning Kagermann & Verteilung integrierter Anwendungen & 1993 & Peter Mertens \\
\hline WI - Aufsatz & Leena Suhl & $\begin{array}{l}\text { Entscheidungsunterstützende Systeme bei der } \\
\text { Produktionsplanung in Fluggesellschaften }\end{array}$ & 1993 & Peter Mertens \\
\hline WI - Aufsatz & Werner Sinzig & $\begin{array}{l}\text { Neue Trends bei der Architektur von } \\
\text { Standardsoftware am Beispiel "Controlling" }\end{array}$ & 1995 & Peter Mertens \\
\hline WI - Aufsatz & $\begin{array}{l}\text { Hans Ulrich Buhl, Volker } \\
\text { Visser, Andreas Will }\end{array}$ & Virtualisierung des Bankgeschäfts & 1999 & Peter Mertens \\
\hline WI - Aufsatz & Georg Disterer & $\begin{array}{l}\text { Individuelle und soziale Barrieren beim } \\
\text { Aufbau von Wissenssammlungen }\end{array}$ & 2000 & Wolfgang König \\
\hline WI - Aufsatz & $\begin{array}{l}\text { Jens Dibbern, } \\
\text { Armin Heinzl }\end{array}$ & $\begin{array}{l}\text { Outsourcing der Informationsverarbeitung im Mittelstand: } \\
\text { Test eines multitheoretischen Kausalmodells }\end{array}$ & 2001 & Hans Ulrich Buhl \\
\hline WI - Aufsatz & $\begin{array}{l}\text { Stefan Holland, } \\
\text { Werner Kießling }\end{array}$ & $\begin{array}{l}\text { User Preference Mining Techniques for } \\
\text { Personalized Applications }\end{array}$ & 2004 & Wolfgang König \\
\hline $\begin{array}{l}\text { WI-State } \\
\text { of the Art }\end{array}$ & Stefan Kirn & Kooperierende intelligente Softwareagenten & 2002 & Peter Buxmann \\
\hline $\begin{array}{l}\text { WI-State } \\
\text { of the Art }\end{array}$ & Arnd Klein, Helmut Krcmar & Electronic Meeting Systems Paradox & 2003 & Peter Buxmann \\
\hline $\begin{array}{l}\text { WI-State } \\
\text { of the Art }\end{array}$ & $\begin{array}{l}\text { Martin Bichler, Alexander } \\
\text { Pikovsky, Thomas Setzer }\end{array}$ & Kombinatorische Auktionen in der betrieblichen Beschaffung & 2005 & Peter Buxmann \\
\hline WI - Schlagwort & $\begin{array}{l}\text { Nicolas Bissantz, } \\
\text { Jürgen Hagedorn }\end{array}$ & Data Mining (Datenmustererkennung) & 1993 & Elmar Sinz \\
\hline WI - Schlagwort & Stephan W. Eder & Grüne Computer & 1994 & Elmar Sinz \\
\hline WI - Schlagwort & $\begin{array}{l}\text { Bernd Jahnke, } \\
\text { Hans-Dieter Groffmann, } \\
\text { Stephan Kruppa }\end{array}$ & On-Line Analytical Processing (OLAP) & 1996 & Elmar Sinz \\
\hline WI - Schlagwort & $\begin{array}{l}\text { Dietrich Cerny, } \\
\text { Hartmut Pohl }\end{array}$ & Trusted Third Parties und Sicherungsinfrastrukturen & 1997 & Elmar Sinz \\
\hline
\end{tabular}

hung der Wirtschaftsinformatik und fragt, welchen Weg das Fach in Zukunft gehen soll. Kenneth L. Kraemer, Rex Chen und Prakul Sharma geben einen fundierten Einblick in Google als einer der erfolgreichsten Internetsuchmaschinen und -werbeplattformen und das Ziel, das weltweit erste Informationsversorgungsunternehmen (information utility) zu werden. Arnold Picot und Oliver Baumann bzw. Matthias Jarke erörtern das Verhältnis zwischen Wirtschaftsinformatik und Betriebswirtschaftslehre bzw. Informatik. Schließlich skizziert August-Wilhelm Scheer die Wirtschaftsinformatik im Spannungsfeld zwischen Wissenschaft und Unternehmertum.

Zwei ausgewählte Beiträge aus der Erstausgabe der Zeitschrift, damals unter dem Namen elektronische datenverarbeitung, aus dem Jahr 1959 verdeutlichen, dass die Notwendigkeit einer integrativen Denkweise - für die heutige Wirtschaftsinformatik ein konstitutives Merkmal - bereits damals in den Köpfen von visionären Vordenkern klar verankert war. So führt John Diebold ein Plädoyer für fundierte universitäre und berufsbegleitende Aus- und Weiterbildungsprogramme im Bereich der Wirtschaftsinformatik. Karl Heinz Kettner fordert den Einsatz von Informations- und Kommunikationstechnologie, um durchgängige integrierte Arbeitsabläufe zu ermöglichen und Unternehmen wettbewerbsfähiger zu machen.

Anschließend finden sich sechs von den (ehemaligen) Geschäftsführenden Herausgebern und Rubrikverantwortlichen ausgewählte beste Beiträge der Rubriken WI - Aufsatz, WI - State of the Art und WI - Schlagwort von 1959 bis 2008. Diese dienen wegen ihrer hervorragenden wissenschaftlichen Qualität, der gelungenen Synthese von wissenschaftlichen Fragestellungen mit deren praktischer Anwendung oder dem frühzeitigen Aufgreifen zukunftsweisender Themen als Vorbilder für künftige Forschungsarbeiten. Aus Platzgründen kann lediglich eine Auswahl abgedruckt werden. Dazu gehören die Beiträge „Betriebsinformatik und Wirtschaftsinformatik als 
notwendige anwendungsbezogene Ergänzung einer allgemeinen Informatik" (Grochla 1969), ,"Verteilung integrierter Anwendungen“ (Kagermann 1993) und "Outsourcing der Informationsverarbeitung im Mittelstand: Test eines multitheoretischen Kausalmodells" (Dibbern u. Heinzl 2001) in der Rubrik WI - Aufsatz, ,,Kombinatorische Auktionen in der betrieblichen Beschaffung" (Bichler et al. 2005) in der Rubrik WI State of the Art sowie "Data Mining (Datenmustererkennung) " (Bissantz u. Hagedorn 1993) und "Grüne Computer" (Eder 1994) in der Rubrik WI - Schlagwort. Eine vollständige Übersicht aller nominierten Beiträge - zwischen denen keine Rangfolge besteht - findet sich in Tab. 1.

Den Abschluss bilden ein Interview von Robert Winter mit Alan R. Hevner zum Thema „Design Science Research for Information Systems" sowie die aktuellen Calls for Papers zu den Heften 1/2010 und 3/2010.

Die eingangs angesprochene Triple-Strategie wirft eine Reihe von Fragen auf. Dazu gehören: Welche Ziele verfolgt die Triple-Strategie? Welche Bestandteile umfasst diese und wie verändert sich dadurch die bisherige WIRTSCHAFTSINFORMATIK? Welche Kernbotschaften wollen wir durch den englischsprachigen Titel vermitteln?

Das primäre Ziel der Triple-Strategie besteht darin, die WIRTSCHAFTSINFORMATIK an den Anforderungen einer sich stärker internationalisierenden Forschung neu auszurichten und für die Zukunft an der Spitze der internationalen Zeitschriftenlandschaft zu positionieren. Gleichzeitig gilt es, die Stärken von Fach und Zeitschrift in die internationale Welt zu transportieren und weiterzuentwickeln, ohne die Verankerung im deutschsprachigen Raum zu vernachlässigen.

- Zwei ausgewiesene Stärken der deutschsprachigen Wirtschaftsinformatik (WI) sind der seit jeher fruchtbare wechselseitige Austausch mit der betrieblichen Praxis sowie der methodenpluralistische, eher gestaltungsorientierte und interdisziplinäre Forschungsansatz. Ersteres zeigt sich insbesondere darin, dass WILehrstühle an vielen wirtschaftswissenschaftlichen Fakultäten die größten und drittmittelstärksten sind. Hier haben wir echte Wettbewerbsvorteile gegenüber unserer Schwesterdisziplin Information Systems (IS): Während die Ergebnisse der IS-Forschung aufgrund ihres stark verhaltensorientierten Ansatzes - der zweifelsohne hohen Rigor-Ansprüchen genügt - in der Praxis oft wenig anwendbar oder teilweise sogar bedeutungslos sind, lösen wir Probleme in der realen unternehmerischen Welt mit hoher Relevanz. Gute wissenschaftliche WIPublikationen erfüllen somit nicht nur das Rigor-, sondern auch das RelevanceKriterium auf hohem Niveau. Sie verfügen über die Praxisnähe, die der IS-Forschung noch fehlt und bereits seit längerem von IS-Kollegen mit Nachdruck gefordert wird (z. B. Davenport u. Markus 1999; Rosemann u. Vessey 2008). Überspitzt könnte man sagen: Ein Großteil der IS-Kollegen forscht - trotz weniger Vorstöße in Richtung Design Science - vermutlich nur noch deswegen nahezu ausschließlich verhaltenswissenschaftlich, weil in einem stark schrumpfenden Markt Lehrstühle nach der Veröffentlichungsleistung vergeben werden und der verhaltensorientierte Ansatz in internationalen Rankings (noch) dominiert.

- Eine weitere Stärke der WI ist die universitäre Ausbildung durch die mittlerweile rund 200 Lehrstühle und Professuren im deutschsprachigen Raum. Durch die interdisziplinäre Ausrichtung an der Schnittstelle zwischen Wissenschaft und Praxis sind WI-Absolventen in gleicher Weise für weiterführende Tätigkeiten in Wissenschaft und Praxis bestens qualifiziert und auf dem Arbeitsmarkt hart umkämpft. Es ist daher keine Seltenheit, dass sich Unternehmen in vielen WI-Studiengängen finanziell und/oder in Form zusätzlicher Lehrveranstaltungen (z. B. Projektseminare) engagieren, um möglichst frühzeitig Kontakte zu späteren Absolventen und Spitzenkräften zu knüpfen. Diese exzellenten Berufsaussichten führen konsequenterweise auch zu einer hohen WI-Nachfrage bei Schulabgängern. Bspw. bewarben sich für den an der Universität Augsburg ab dem Wintersemester 2008/2009 eingeführten WI-Bachelor-Studiengang ca. 7 Schulabgänger pro Studienplatz. Im Allgemeinen sind die WI-Einschreibungszahlen seit mehreren Jahren konstant und wachsen sogar moderat. Im Gegensatz dazu verzeichnen die IS-Einschreibungszahlen - bis auf wenige Ausnahmen - in den letzten Jahren einen massiven Einbruch und sanken allein zwischen 2000 und 2006 um durchschnittlich ca. $75 \%$ (Nickerson 2007). Zum anderen beinhalten laut einer aktuellen Studie derzeit nur noch 50 \% der führenden nordamerika- 
nischen Business Schools in ihren MBA-Programmen Pflichtkurse im Bereich (Management) Information Systems (Navarro 2008). Die WI hat daher allen Grund, nicht nur am Abbau ihrer Schwächen zu arbeiten, sondern ihre Stärken selbstbewusst weiter auszubauen und diese keineswegs auf dem Altar einer falsch verstandenen internationalen Anpassung zu opfern.

- Eine Stärke der Zeitschrift ist ihr doppelt-blinder - was z. B. für die internationale Spitzen-Zeitschrift Information Systems Research erst seit März 2008 zum Standard gehört (Sambamurthy 2008) - sowie konstruktiver und zügiger Begutachtungsprozess. Jeder Beitrag wird durch den Geschäftsführenden Herausgeber mit Unterstützung durch sehr kompetente Fachkollegen vorbegutachtet und dann - in der Regel nach einer ersten Überarbeitung - von mindestens drei internationalen Experten aus Wissenschaft und Praxis (davon normalerweise zwei aus der Wissenschaft und einer aus der Praxis) sofern Erfolg versprechend über mehrere Runden bis zur Publikationsreife weiterentwickelt. Die Einbindung von Praxis-Gutachtern zeigt auch hier den Stellenwert des Relevance-Kriteriums. Es ist unser Bestreben, im Sinne von "journal editors as diamond-cutters rather than gatekeepers" (Straub 2008, S. vii) durch konstruktive Anregungen den aus wissenschaftlicher Sicht interessanten Kern herauszuschälen und für die Community wertvolle Beiträge zu identifizieren. Dadurch vermeiden wir die von Detmar W. Straub (2008) und Carol Saunders, derzeitiger und ehemalige Editors-in-Chief der MISQ, diskutierten Fehler vom Typ II („Ablehnung eines guten Beitrags"). Zudem benötigen wir - im Gegensatz zu anderen Spitzen-Zeitschriften - dank des Engagements der ehemaligen Geschäftsführenden Herausgeber und des gesamten Herausgeberkreises in aller Regel weniger als zwei Monate für eine Begutachtungsrunde. Dadurch nehmen wir den Autoren Unsicherheit und setzen Anreize für eine kumulative Forschung mit guter und konstruktiver Interaktion. Beiträge werden nur dann abgelehnt, wenn absehbar ist, dass diese trotz Vorbegutachtung auch nach mehreren Überarbeitungsrunden den Ansprüchen der Zeitschrift an Rigor und Relevance nicht genügen werden. Das ist leider bei mehr als $70 \%$ der Einreichungen der Fall. Dennoch erhalten wir auch bei Ablehnungen sehr häufig positive Rückmeldungen bzgl. unseres konstruktiven und zügigen Begutachtungsprozesses, was letztendlich - selbst wenn die Beiträge in einer anderen Zeitschrift veröffentlicht werden - dem Fach nützt.

Um diese Ziele zu erreichen sowie die Stärken weiter auszubauen und international bekannt zu machen, besteht die Triple-Strategie aus drei Zeitschriften:WIRTSCHAFTSINFORMATIK, BISE und WUM.

- Die bisherige WIRTSCHAFTSINFORMATIK erscheint weiterhin wie gewohnt zweimonatlich mit Schwerpunktheften und multithematischen Heften als Printausgabe. Im Zuge der stärkeren wissenschaftlichen Ausrichtung konzentriert sich ihr Inhalt ab diesem Heft auf die wissenschaftlichen Rubriken WI - Aufsatz und WI - State of the Art sowie auf die Rubriken WI - Interview, WI - Meinung/Dialog und WI - Schlagwort, die auch für internationale Forscher attraktiv sind. Die anderen bisherigen Rubriken finden sich entweder auf unserem Internetportal http://www.wirtschaftsinformatik.de und/oder migrieren leicht angepasst in die WUM.

- Die BISE erscheint ab diesem Heft parallel und inhaltlich identisch - im Sinne einer Eins-zu-eins-Übersetzung - zur deutschsprachigen Ausgabe. Die Zeitschrift ist als E-Journal konzipiert und - neben http://www.wirtschaftsinforma tik.de und http://www.bise-journal.org - über SpringerLink (http://www. springerlink.com), der weltweit größten Online-Bibliothek für Wissenschaft, Technik und Medizin, beziehbar. Die Marktanteile von SpringerLink haben sich in den letzten Jahren derart positiv entwickelt, dass der überwiegende Teil der internationalen Forscher in den meisten Ländern, wie z. B. den Vereinigten Staaten, Kanada, Australien, Skandinavien und Osteuropa, von ihren Universitäten bzw. Forschungseinrichtungen aus bereits heute unkompliziert auf BISE zugreifen kann. Um die Zeitschrift möglichst schnell bekannt zu machen, wird diese für diese Erstausgabe mit einer sehr hohen - und danach kleinen - Printauflage erscheinen, die an nationale und internationale Multiplikatoren gestreut wird. Zur verstärkten internationalen Ausrichtung des Herausgeberkreises 
konnten wir bereits einige herausragende internationale Kollegen gewinnen, die wir in der Rubrik WI - In eigener Sache vorstellen. Beiträge zu beiden wissenschaftlichen Zeitschriften können nach wie vor in deutscher und englischer Sprache eingereicht werden. Sie werden bei Annahme in enger Abstimmung mit den Autoren von einem kompetenten Übersetzerteam in die jeweils andere Sprache übersetzt, erscheinen somit stets zweisprachig und haben eine wesentlich höhere internationale Sichtbarkeit als bisher.

- Die WUM - deren Erstausgabe ebenfalls zusammen mit diesem Jubiläumsheft erscheint - bildet mit einer eigenen beim Gabler-Verlag angesiedelten Redaktion und einem mit hochkarätigen Wissenschaftlern und Praktikern besetzten Advisory Board das Bindeglied zu den deutschsprachigen Wirtschaftsinformatik-Praktikern. Ziel ist es, diese nach dem Vorbild der Harvard Business Review noch besser als bisher mit inhaltlich fundierten, professionell und magazinartig aufbereiteten und gleichzeitig in höchstem Maße praxisrelevanten Informationen zu versorgen. Zu diesem Zweck gibt es eigenständige und aktuelle redaktionelle Beiträge sowie praxisorientiert aufbereitete Management Summarys ausgewählter angenommener Beiträge der wissenschaftlichen Zeitschriften mit Hinweisen auf die Originalpublikationen.

Gleich welche Zeitschrift ein Leser bezieht: Jeder Abonnent einer der drei Zeitschriften hat über unser neu gestaltetes Internetportal (http://www.wirtschaftsin formatik.de bzw. http://www.bise-journal.org) vollständigen Zugriff auf unseren gesamten deutsch- und englischsprachigen Inhalt und natürlich die Online-Archive aller drei Zeitschriften.

Eine wichtige Frage im Rahmen der Triple-Strategie war die nach dem adäquaten Titel der englischsprachigen Ausgabe und der damit verbundenen Botschaften. Mit "Business \& Information Systems Engineering (BISE)" transportieren wir zum einen den bereits diskutierten gestaltungsorientierten Ansatz über den Titelbestandteil „Engineering". Zum anderen signalisieren wir, dass wir sämtliche technoökonomisch orientierten Leser und Autoren als Zielgruppen ansprechen. Durch "Business" sprechen wir Kollegen an, die wirtschaftsinformatische Fragestellungen aus einer eher betriebswirtschaftlichen Perspektive untersuchen. Durch "Information Systems" adressieren wir die wachsende Zahl der an Rigor und Relevance interessierten internationalen Kollegen unserer Schwesterdisziplin. Durch „Engineering“ erreichen wir zudem die deutschsprachigen Wirtschaftsingenieure und die englischsprachigen Industrial Engineers, die sich mit Wirtschaftsinformatik-nahen Themen auseinandersetzen; genauso einschlägige Kollegen aus der praktischen Informatik. Die mehrdeutige Lesart des Titels adressiert also betriebswirtschaftlich (Business bzw. Business Engineering), wirtschaftsinformatisch (Information Systems bzw. Information Systems Engineering) und ingenieurswissenschaftlich (Business Engineering bzw. Information Systems Engineering) orientierte Leser und Autoren, die Schnittstellen zwischen den Disziplinen und somit die gesamte technoökonomisch orientierte wissenschaftliche Community.

Zusammenfassend lässt sich sagen: Durch die Triade aus WIRTSCHAFSTINFORMATIK, BISE und WUM erreichen wir die deutschsprachige Wirtschaftsinformatik-Community in Praxis und Wissenschaft noch besser als bisher sowie gleichzeitig die gesamte internationale technoökonomisch orientierte wissenschaftliche Community!

Im Namen aller ehemaligen Geschäftsführenden Herausgeber und Herausgeber dieses Jubiläumshefts - den Kollegen Hasenkamp, König, Mertens und Szyperski sowie im Namen des gesamten Herausgeberkreises danke ich allen Autoren, die durch ihre hervorragenden Beiträge geholfen haben, ein dem 50. Geburtstag der WIRTSCHAFTSINFORMATIK und dem Startschuss der Triple-Strategie in gleicher Weise angemessenes Heft zu gestalten. Ebenso danken wir den Gutachtern, welche die Beiträge fachlich sehr kompetent über mehrere Runden betreut haben. Beiden Gruppen danken wir zudem für die Geduld und das Verständnis, mit der sie sich mit den eingeforderten Überarbeitungen und unseren straffen Zeitrestriktionen auseinandergesetzt haben. Auch der Redaktion - insbesondere auch wegen der Unterstützung bei der Übersetzung - und dem Verlag gebührt ein großer Dank für die erhebliche Mehrarbeit. Ich danke auch Robert Winter, der als verantwortlicher Mitheraus- 
geber die Triple-Strategie von Anfang an entscheidend mitgeprägt hat sowie u. a. mehrere Partnerfirmen und internationale Herausgeber gewinnen konnte. Vielen Dank ebenfalls an alle Autoren, Gutachter und Herausgeber der letzten 50 Jahre, ohne die die WIRTSCHAFTSINFORMATIK ihre Erfolge nicht in dieser Weise hätte feiern können.

Ein ganz besonderer Dank - im Namen des Herausgeberkreises und des Verlags ergeht an unsere Partnerfirmen Allianz, Commerzbank, IBM, McKinsey, SAP und TLabs, die durch ihre großzügige finanzielle Unterstützung dieses Jubiläumsheft und die Triple-Strategie ermöglicht haben.

Unser letztes Wort richten wir an die Community: Die Erfolge der letzten 50 Jahre im deutschsprachigen Raum waren nur wegen des Engagements aller Mitglieder möglich. Bei der Triple-Strategie verhält es sich genauso. Wir bitten daher jeden von Ihnen, die Triple-Strategie mit uns positiv zu entwickeln, diese im persönlichen Netzwerk zu multiplizieren und dadurch bekannt zu machen. Nur so werden wir auch in Zukunft national und international erfolgreich sein!

Prof. Dr. Hans Ulrich Buhl

\section{Literatur}

Davenport TH, Markus LM (1999) Rigor vs. relevance revisited: response to Benbasat and Zmud. MIS Quarterly 23(1):19-23

Navarro P (2008) The MBA core curricula of top-ranked U.S. business schools: a study in failure.

The Academy of Management Learning and Education 7(1):108-123

Nickerson RC (2007) IS enrollment decline. Summary of ISWorld responses. http://online.sfsu.edu/ rnick/isenroll.pdf. Abruf am 2008-09-04

o. V. (1959) Elektronische Datenverarbeitung. Fachberichte über programmgesteuerte Maschinen und ihre Anwendung. elektronische datenverarbeitung 1(1):3-4

Rosemann M, Vessey I (2008) Toward improving the relevance of information systems research to practice: the role of applicability checks. MIS Quarterly 32(1):1-22

Sambamurthy V (2008) Editorial note. Information Systems Research 19(2):125

Straub DW (2008) Thirty years of service to the IS profession: time for renewal at MISQ? MIS Quarterly 32(1):iii-viii 


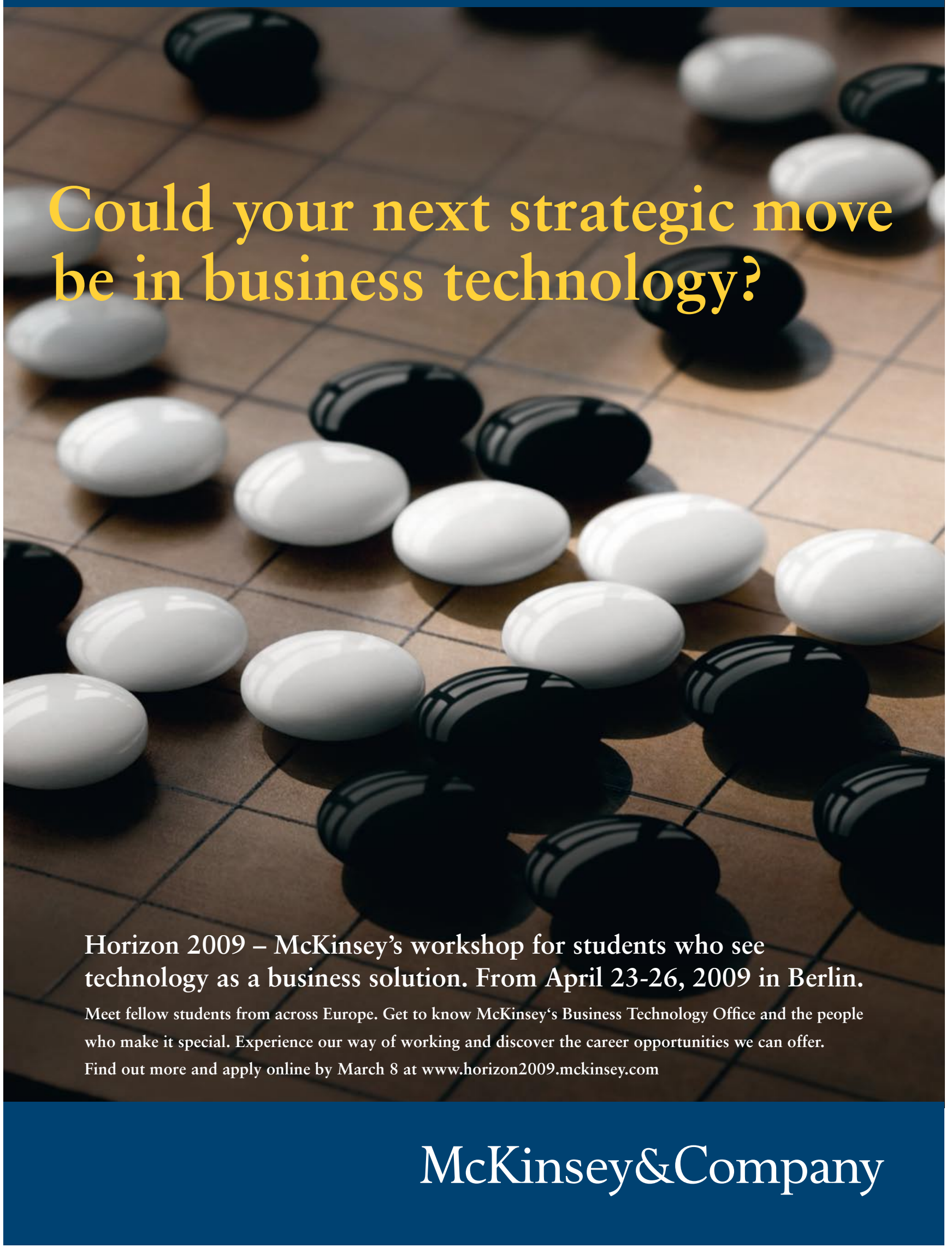

